\title{
Containing Family Clutter
}

\author{
Laurel Swan', Alex S. Taylor ${ }^{2}$, Shahram Izadi ${ }^{2}$ and Richard Harper ${ }^{2}$ \\ 1 School of IS, Computing and Mathematics, Brunel University, UK \\ 2 Microsoft Research, Cambridge, UK
}

\begin{abstract}
In this paper, we present material from an ongoing ethnographic investigation of family life. Drawing on selected fieldwork materials, we look at the ways families deal with household clutter, and in particular how clutter can be contained in bowls and drawers. Based on this research, a case is made for rethinking digital media management in domestic settings. We argue that existing solutions, largely based around the PC, inhibit the casual storage and loose organization of content, properties afforded in both bowls and drawers. We explore a design perspective that aims to address this by building on physical properties of the bowl, using salient properties from fieldwork material to sketch out an early concept of an augmented bowl designed to hold physical and digital content.
\end{abstract}

\section{Introduction}

Where there is order, where things are classified and given a rightful place, there will always be matter out of place, always disorder, always mess [see 7]. In our ordered worlds, clutter is ever-present, and nowhere more so than in the family home: letters arrive on the doormat, children come home and throw their things on the floor, dirt accumulates and the laundry builds up. Chaos looms.

Our central argument in this paper hinges on the way families deal with clutter. For most families, the ideal of the perfectly ordered house sits uneasily with the reality of family life, and a constant balance must be negotiated between the family members assorted paraphernalia and a 'tidy enough' house. Families, of course, do not always systematically maintain this balance and often resort to intermediary solutions to deal with the resultant clutter. Using materials from an ethnographic study of family homes, we will argue that this is a practical, commonplace approach to everyday life at home and will show how containers, such as bowls and drawers, help enable this economy of living. 
Using this as a basis, we aim to explore an alternative perspective to managing and arranging digital media. We consider how new digital technologies are adding to the proliferation of stuff in homes and yet, by and large, fail to assist in the containment of clutter. Instead, the digital realm offers increasingly sophisticated solutions for managing and organizing media that require prolonged and sustained interaction. These tools allow family members to archive images, edit video, create music compilations and much else besides, but often, indeed very often, these tools demand more time and effort than is available [12]. Of course, people do sometimes want to spend time and effort organizing, archiving and editing digital media; however, due to the constraints and demands of family life, this is not always possible and the design of current digital technologies does not allow for this variability of effort.

In the following, we will begin by touching on the main themes of $\mathrm{HCI}$ research related to domestic technology and consider these with respect to the minimal effort given to managing household clutter. We then detail materials selected from our ongoing ethnographic investigation of family homes. We foreground the casual ways that families store and loosely organize materials, and draw specific attention to the at-handedness, visible function and flexibility of physical containers such as bowls and drawers. We discuss how these containers afford minimal effort to contain clutter of particular kinds: items en route to somewhere else, items with a limited life span, items where their sentimental character makes disposal problematic, or items that no one knows what to do with and therefore have no 'proper' home.

Drawing on this material, we will then discuss the implications of our analysis for the management of digital media solutions in the home. In referring to digital media, we confine ourselves to content held on devices like mobile phones, digital cameras and music players, as opposed to materials generated by email and the internet. Finally, to address design directly, we outline some design ideas we have been exploring to deal with digital media in lightweight ways. Our ideas are encapsulated in a presented design sketch, in which we investigate how the properties of a physical bowl might be augmented. Although it might appear an unlikely candidate for research, our hope is for the bowl, as an idea, to illustrate how we might think about designing for the casual, low effort practices we use to manage clutter in the home.

\subsection{Household Clutter in HCI}

In much of the research surrounding the home in $\mathrm{HCl}$ and its related design fields, little (if any) attention has been given to the general problem of untidiness and clutter. Despite its all too familiar presence in home life, the domestic environment appears sanitized, clean, and clutter-free. For example, where technological concerns have been paramount, as in the ongoing and widely dispersed Smart Home Programme and select areas of Ubicomp, much attention has been given to the use of and interaction with sensors [13], networked appliances [2] and home automation [19]. Other efforts have been concerned with monitoring, both of security [3] and the health of a home's inhabitants [14]. While laudable for other reasons, these projects are vuinerable to the oft-made criticism from the ethnomethodological perspective, a 
criticism that holds that the 'work' or effort routinely put in to making places like the home unique is regularly rendered invisible in technological visions [e.g. 20]. Ignored in such projects, for instance, is the considerable work required to keep the home in order $[1,22]$ and, relevant to the evidence presented here, the efforts and resources enlisted to keep clutter at bay.

More detailed investigations of home life have partially addressed such failings $[5,11,23]$. These works have not however placed direct attention on clutter per se nor the tools or behaviors used to manage it. Rodden et al. [17], for example, have discussed the role of 'stuff' as an important component of designing technology for domestic settings. However, their 'stuff of the home' tends to be of a purposeful, functional nature, whereas the stuff in the households we examine is occasionally interacted with, but more often disregarded, left to coalesce in hidden places. Similarly detailed investigations into home life have looked at the collaborative aspects of practical activities such as TV viewing [16,21] and photo sharing $[6,9]$. While such studies pay heed to activities that take up considerable time and thought, they again remain concerned with a discrete, purposeful engagement with the home environment. The practices surrounding the containment of household clutter are less purposeful or discrete but are, we would argue, more pervasive, ubiquitous and indeed continuous.

\section{Fieldwork}

The data we present to explore these arguments are drawn from an ongoing, ethnographic study of family life. They have been chosen from a larger corpus of field investigations, spanning 18 months, with 12 families living in the UK. Excerpts from interviews and observations with three mothers constitute the core of the materials used. This limited but focused explication will illustrate some of the rich and varied ways in which homes and the stuff within them are organized.

\subsection{Effortless containment}

To begin with, we consider the following from Nicola, the mother of two sons, aged six and ten. In her open plan kitchen/dining room, three stacks of bowls sit near the work surfaces, seemingly ready to prepare or serve food. It is immediately apparent, however, that they have been appropriated for an assortment of bits and pieces. Here she describes the origin of this usage:

I suppose it's because you have stuff [said with emphasis in a pejorative tone] and you need to put it somewhere and bowls seem quite a good receptacle in that they just swallow everything up. Ummm,... [pauses] completely without any thinking or planning... (see Fig. 1) 


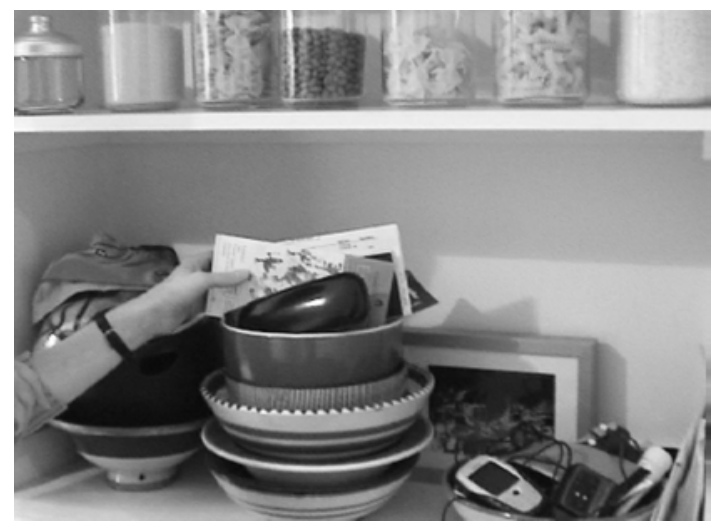

Figure 1. Nicola's three bowls.

The stuff Nicola refers to is a seemingly haphazard mixture of sunblock, cheque stubs, door locks, mobile phones, and much else besides. In an attempt to keep clutter at bay, Nicola has appropriated a class of common household items, bowls, to act as repositories for this varied paraphernalia. The ability of bowls to 'swallow everything up' makes them well-suited to containing the family's flotsam and jetsam with a minimum of effort. As we shall see, this technique has become part of the organization of her home. She continues, describing her husband's use of bowls:

... sometimes he'll plug into them. So he knows for example that- it's never talked about, but he'll know that batteries go in that bowl, keys go in that bowl and, if you have paper work that needs sorting, it'll go in that little pile. So I guess he tunes into it almost subconsciously. They are my systems, but they become the home systems I suppose. And they're really not- it's rather a grand word to call them systems actually.

On the face of it, then, we see that bowls and piles are considered useful in a home because they help family members achieve, in an ad hoc, lightweight way, some semblance of order with minimal effort. As our next excerpt illustrates, however, the easy-to-hand use of bowls on Nicola's part is not a wholly satisfying system. Although she refers to piles in the following excerpt, Nicola's remarks are directed at the general clutter on show in her house:

I think a file, somehow, would just get forgotten about more than just a visible pile that's actually irritating me. That's part of it. Part of it is that I don't like clutter, even though you wouldn't know it [gestures around house]. I don't like all these piles of things everywhere so if I deliberately make a pile then it's sort of a motivation to get rid of it as well.

Unlike things that are filed away, visible piles and bowls full of stuff attract attention, reminiscent of the things we leave out to trip over to act as reminders [15]. Nicola's bowls summon attention because householders can see at a glance that things are in them, waiting to be 'properly sorted'. For Nicola, the bowl serves not only as a to-hand solution, but also stands as a slightly irritating reminder of the tidying and organizing that needs to be done. As such, they serve as a partial or temporary solution to the problem of tidying up, sufficient in the short term.

Another of Nicola's bowls, this one tucked out of sight behind the kitchen door, raises another important property, that of layering. Pointing to the bowl, Nicola runs through its contents: 
This is old mobile phones that we're going to chuck out but I think actually I'll get them recycled somehow. Film for the camera, batteries- the inevitable batteries because if you have kids all their toys need batteries, the A-to-Z. You know, sort of bits and pieces but if you dig down to the bottom I'm sure there are things in there that I have long since forgotten about. So it isn't very organized in that respect. The things on the surface are important, but in some sense it's like geology.

Nicola's suggestion that there are things long since forgotten about under the surface and her reference to geology conjures up the sense of excavation common to clutter containers. Stuff is thrown in to the bowls in a way that is almost without thought and the layering that results is achieved because of the bowl's depth and its sidedness. Items are kept together in ways we are immediately familiar with; newer items remain on the surface whereas the older and/or smaller content seep towards the bowl's sediment. The physical layering thus becomes a queue for recollection, management, and for navigation through the accumulated layers.

\subsection{Types of clutter}

Our next examples examine the myriad forms clutter can take and how placing clutter out of sight or having it hidden can serve quite particular purposes. Emma has a 'junk drawer' with a broken front, located amongst the kitchen cabinets (Fig. 2). In contrast to Nicola's piles and bowls, Emma's drawer is not particularly noticeable; when closed, it looks like any drawer, save for its broken front. Inside the drawer is a jumble of string, spare plugs, cards, sunglasses and bicycle lights. There is no explicit system of separation or organization; it simply appears to be clutter. Emma's description, however, reveals that there is some form of order to the drawer, albeit a loose one:

This is where I just put things where I- you know where you think you really want to throw it away but you don't feel that you can... so it's a combination of those things and little things that I don't have a home for but I should have a home for, like the tape measure, and the rulers, and the paper clips, and things.

Emma's description of the things "you really want to throw away but you don't feel you can", conveys some of the essence of clutter. Her use of the word 'home' regarding the paper clips and rulers is also telling; it gives a sense of things having a right and proper place. Presumably these little household tools deserve a 'home' due to their usefulness and ubiquity, but paradoxically their 'home' ends up amongst the homeless, in the clutter.

Digging deeper, it emerges that there is further categorization within the clutter. Emma elaborates while sorting the contents into several small piles:

Those are dice, but again they should go, there's a little bag we have upstairs for dice so that should be, they should all be in the dice bag... Lego, that needs to go in the Lego box...more dice, they should all go in the dice bit...

In Emma's drawer are items from various larger collections stored elsewhere in the house. This is a particular type of clutter; it is "matter out of place" [7]. These pieces do have a 'home', they are simply not in it, and Emma's description of them "going places" gives the sense that they are in transit, albeit perhaps temporarily on a stopover in the junk drawer. 


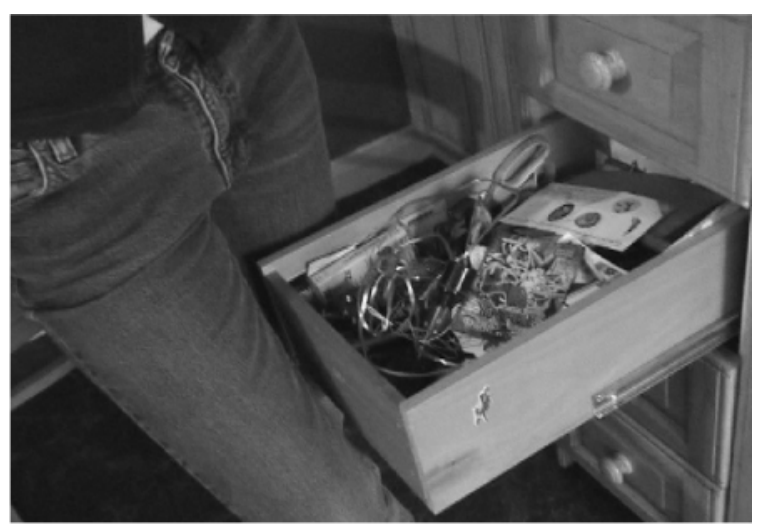

Figure 2. Emma's junk drawer.

Of course, it is not always so clear where items of clutter belong or what status they hold. While adding things to a pile she has designated rubbish, Emma holds up a card, explaining that it was from the last Harry Potter movie and "was terribly precious for a short period of time". The fact that a sizable proportion of things in junk drawers can be disposed of upon sorting out is interesting. It suggests that items can go into the drawer with one status and come out with another, that something "terribly precious" can transform into rubbish within the drawer. Items such as media associated with new movies derive some of their value from their newness; when the novelty wears off, their status plummets. The drawer can thus act as a holding place, a safe spot for things with a temporary shelf life to live until that life expires.

In the next excerpt we see that clutter bowls and junk drawers can function as repositories for items with problematic status:

...that's an air freshener for a car that [smells the wooden apple shaped object]- err, smells horrible, but it was in the car when we bought it so we've hung onto it for sentimental reasons... when we bought our car that we have now it was new and for some reason it was in there. I don't know why it was just- I think the kids thought it was exciting that it came with an apple as well. You know 'new car and wooden apple!' [laughs] so for some reason we still have it.

Although Emma's drawer might not, on first glance, seem an obvious place for archiving memorabilia, her wooden apple raises an interesting point. Namely, not all sentimental objects are guaranteed a place on the mantelpiece, as it were, and junk drawers and bowls give refuge to items of ambiguous value.

\subsection{Battling clutter}

We want to build on this idea of ambiguity in our final example, taken from an interview with Olivia, mother to two girls, aged six and nine. Olivia's family home is especially tidy, and on first glance does not seem to be a promising arena for studying clutter. There is none visible, and Olivia herself claims that she does not keep "stuff" and throws away as much as she can. Delving further, however, we are able to find little hints of clutter, and more interestingly, Olivia's efforts to keep them at bay. Tucked into the corner of a cupboard of wine glasses is a small bottle of 
homeopathic drops. The bottle of ointment, a gift from her daughter to help her (the mother) to unwind, presents a small problem of classification for Olivia:

I thought well- I couldn't think where to put it actually, and you can't see it when you close the door... I mean really it could be put in another drawer. But this is me, I think to myself 'Why is that out? Put it away.' So it may not be in the right place but I put it away because I can't stand stuff lying about.

Although Olivia's gift is placed out of sight in a cupboard, we discover it does in fact have its right and proper place. The overriding criteria appears to be that stuff cannot be left lying about and therefore a bottle meant for a drawer is put away elsewhere. Unbeknownst to her daughter, stress is relieved not by using its content but by hiding the bottle from view.

Olivia has recently had her kitchen and adjoining utility room redesigned with banks of closets, cupboards and drawers, and feels that she now has "places to put stuff". Peering into various drawers, we find collections of like things, neatly separated by containers, dividers, trays, plastic bags, etc. Indeed, the drawers are the epitome of organization, and it does seem as if Olivia has perhaps eradicated the specter of clutter by categorizing it to the $n^{\text {th }}$ degree in all its minutiae. In a drawer of tools and household implements, however, we get a glimpse that all is not as it seems. This drawer has, besides a tray full of tools and a case of socket wrenches, a biscuit tin of batteries and a plastic tub of keys. Olivia explains that the tub is specifically for keys, separated out into a plastic bag, and keyrings. When the number of keys is remarked upon, she replies: "I have no idea what they're for, but I've kept them because that's where they go."

Olivia has thus taken something of uncertain status found in nearly every household - in this case, keys to unknown locks - and has given them a home. In doing so, she has not resolved their status; she has no more idea of their rightful destination than either Nicola or Emma would, but by giving them a designated place "where they go" she has organized them and attended to them, compartmentalizing and thereby minimizing what one might call their ambiguity.

Exploring further in the key tub, we find several un-key-like items. Olivia's response is illuminating:

They're just things, aren't they? I don't know what to do with them so I put them in here... [pointing to a glass sphere]. That's a ball off the garden swing. It's of absolutely no use but it's beautiful so I couldn't throw it away, could I? So I've put it in here.

In the glass ball off the garden swing, there are echoes of Emma's wooden apple car freshener, of an item having the dual status of being junk and sentimental at the same time. Although Olivia tackles the business of classifying the miscellany of the household with fervor, and has closets, cupboards, drawers and dividers to help her, ultimately she too ends up with a small tub somewhere full of 'just things'.

\subsection{Containing disorder}

Reflecting on the prior examples-Emma's drawers, Olivia's categorisations and Nicola's bowls and piles - we see that the containers and the material within have a number of distinctive properties. Containers that demand low levels of interaction, like bowls and drawers, allow our three households to deal with clutter with minimal 
effort. These devices afford at-handedness; their placement near to where clutter accumulates offers a simple and lightweight resource for containing or hiding things. Moreover, bowls and drawers allow the placement of things in them without careful thought or deliberation. Part of their success is due to their visible functionalityanyone can see what they are for, no labeling is needed and no expert training is required before tossing something in; as we have seen, even husbands know how to use them. The intelligibility of these devices is further represented by their layers and the 'geology' of the stuff they contain, both serving to show the history of their use.

\section{Managing digital media in the home}

In the remains of this paper, we wish to contemplate how these practices associated with managing clutter in the home might be applied to the digital realm. To develop our ideas we have chosen to focus on digital media such as digital photos, music and video, even though the fieldwork materials described above could of course be applied to a variety of digital technologies. This is in part due to the increasing adoption of digital media players and recorders in the home, a trend that suggests digital content will be entering and staying in our homes in ever-greater quantities. More importantly, What intrigues us about the currently available software solutions for managing digital photos, video, music, etc. is that they retain a fairly conservative perspective on organization, one that arguably overlooks the kinds of practices we believe hold an important, if not elemental place in the home.

\subsection{Digital media on the PC}

Before reflecting on our own design explorations, we want to briefly consider the $\mathrm{PC}$, probably the most commonly used solution for storing, managing and organizing digital media. By reflecting upon the use of the $\mathrm{PC}$, we hope to provide a clearer position on some of our ideas that follow.

The PC provides the necessary hardware and software to perform a host of functions on diverse media formats such as digital photos, video, music and so on. However, this PC-centric model of handling digital media contrasts with the minimal effort practices and casual storage afforded by bowls and drawers. As presented in the fieldwork, bowls and drawers function as a lightweight method for holding content, one that is readily adopted in the home. Bowls and drawers store and loosely organize as a natural consequence of placing objects within them, and therein lies their appeal; very little if any effort is required for bowls to work in an intelligible way. Indeed, it is the limited rather than abundant number of features that make it compelling.

In contrast, the PC's ability to perform a range of activities related to storage, organization and manipulation demands a level of complexity that makes it unwieldy and thus difficult to incorporate into everyday routines. Because of this complexity, no casual way exists to simply contain or store digital media - there is no equivalent to an object simply placed in a bowl or drawer and minimally organized by its size and when it was placed there. There is an in-built formality to both containing (or 
'uploading') content and organizing it on the PC that is manifestly not the case with physical containers. Consequently, the PC is better suited to the more formal storage and organization of content that requires highly focused interaction, the sorts of activities that are put off in the family home for more time-bounded and infrequent occasions.

\subsection{Digital media containers}

To contemplate this problem and consider the practical issues, we have started to sketch out a conceptual design space. The primary motivation underlying our initial design forays has been to support the casual and informal organization of digital media by providing lightweight methods of interaction that are intelligible to the user. The practical focus thus far has been on augmenting a physical bowl in order to explore some of the empirical ideas above and to use this exercise to draw lessons for further design iterations.

The current design 'sketch' (only partially implemented) is based around a semitransparent physical bowl capable of holding both digital and physical content (Fig. 3 ). The basic idea is that when devices such as cell phones and digital cameras are placed inside the bowl, their content is copied and displayed on the bowl's sides. As more content is added, existing items fall deeper to the bottom. Similar to the bowls in our fieldwork, our augmented bowl becomes a temporary holding place where digital content can be casually added and viewed, and loosely arranged, before later, maybe much later, finding a place elsewhere.
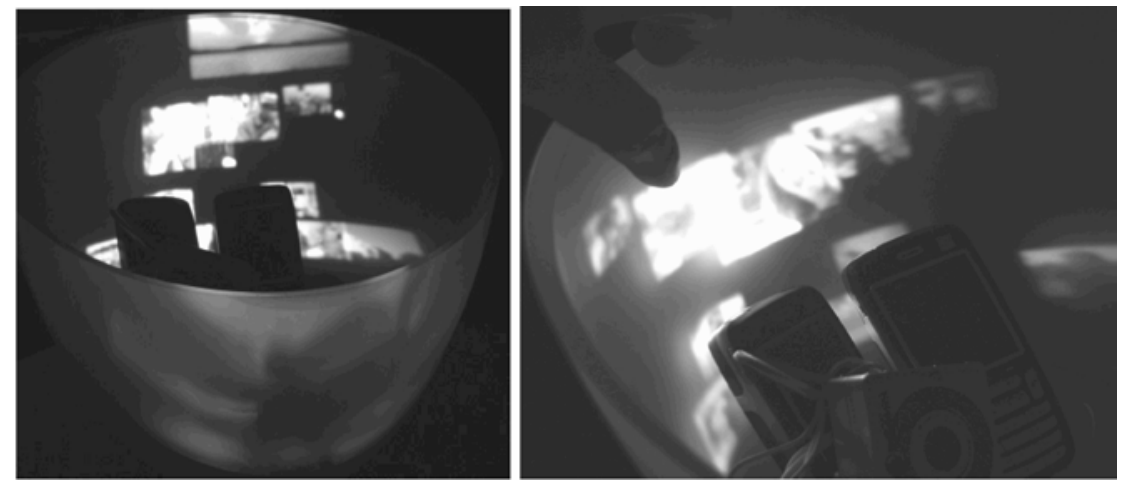

Figure 3. Bowl sketch built using projection system and translucent glass bowl.

\section{Containment}

In our early mock-ups of the above sketch, a primary motivation has been to support the minimal effort qualities of clutter containers. We wanted the simple act of placing a device in the bowl to accomplish the containment of digital as well as physical media. One way in which we aimed to do this was by preserving the familiar, physical properties of a bowl and thus retaining the intelligible and lightweight interactions that are afforded. In short (and echoing Nicola's comments 
above), we used a bowl shape to exploit the properties bowls exhibit, properties that allow people to "tune" into their use "almost subconsciously".

For similar reasons, we wanted the bowl to have only limited functionality. The limited features that were chosen to facilitate informal and casual use that would, again, be intelligible. For example, we imagined that image thumbnails might be moved by simply moving the associated device in the bowl or alternatively by simply interacting with the thumbnails directly. Moreover, the projected thumbnails would be 'stretched' as they were moved from the rounded bottom of the bowl to its relatively flat sides (see Fig. 4). This 'stretching' was seen to exaggerate the effect that would be obtained by moving a projected image from a tightly curved to flat surface: a visible and intelligible property of the bowl's physical form.
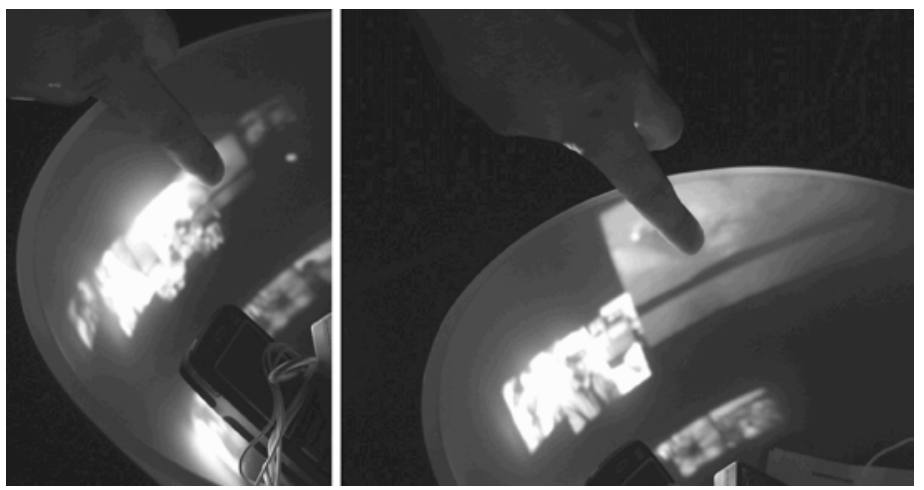

Figure 4. Thumbnail stretching.

\section{Storage}

It is, we have suggested, the simplicity of use and to-handedness that promote bowls as particular sorts of storage receptacles. As we have seen in contrasting the PC with physical containers, items can be literally tossed into bowls and drawers with minimal expectation of order. Like we saw with Nicola and her home's bowls, and even Emma's dedicated junk draw - in her kitchen - this minimal organization of household stuff into containers is routinely bound up with where it can coalesce and how it interleaves with a household's comings and goings. We have aimed to build on this by designing the augmented bowl to operate as a standalone container, with a home having the choice to place the bowls in different locations.

Contemplating the individual storage capabilities of these augmented bowls, it is evident the size of the bowl clearly restricts the extent to which media can be organized. The point in exploring the bowl as a digital media container, however, has been precisely to limit the organizing capabilities. Our aim has been to explore a just-good-enough solution for loosely storing grouped items (as opposed to assuming that increasing the storage capacity is automatically preferable). Next, in introducing the idea of surface ecologies, we will comment on how we see a more involved and explicit organization of media might be accomplished.

We have considered in our designs how some media may be obscured as more items are added. Rather than a limitation, we see this to be an intelligible feature: as 
with a conventional bowl, as items are placed in it, other content is obscured. To view lower lying items, the top layers must be sifted through, moved apart or removed. In terms of detaching media from its associated device, we have tried to maintain the idea of layering that both Nicola and Emma referred to where newer items remain atop a bowl's content and older items end up, over time, constituting a container's sediment. In our design, content can be 'peeled' away from its device and left on the top 'digital' layer until obscured by other content. To detach the media, a device's thumbnails are held down in the bowl with a finger as the device is pulled away. In general, we have sought to explore is how properties such as fullness, layering, and loose temporal ordering might hold true for a digital container such as the augmented bowl.

\section{Surface ecologies}

Arguably, other augmented surfaces, such as tabletops and walls, offer a technically more feasible solution to the problem of digital media containment and storage. The difficulty involved in projecting onto and detecting interactions with tabletops and walls has been subject to extensive research. What is evident from our observations is that tables and walls do not lend themselves to the same types of containment and storage functions afforded by bowls and drawers. This point is best illustrated by example. Consider how horizontal surfaces such as tables play into the patterns of home life. As we and others have observed, tables are ideally suited to the display and organization of materials, particularly with collaborative activities; the physical nature of the table lends itself to having content spread over it and people arranged around it for the purposes of sorting, organizing, viewing, playing, eating and so on. Usage is thus driven by bounded activities, something all the more pertinent in the family home where table-use is regulated by a household's daily rhythms and negotiated by family members. Any containment afforded by tables is consequently constrained by who and what has overall rights to the table. The table has a social as well as physical character in the home that means any storage is time limited and bound by an established social order.

Bowls, in contrast, serve a very different function. We have suggested that one of the reasons why clutter bowls and drawers exist in the home is to keep disorder at bay, to contain and store it, sometimes out of sight. Tables and walls place clutter on show, revealing to others our house's disorder and possibly worse, acting as a reminder of our own idleness. As Olivia demonstrates in placing her bottles of ointment out of sight, the-need-to-put-away can be a moral imperative.

A sensitivity to the actions and activities afforded by tabletops, walls, containers, etc. gives an indication of how bowls might operate within the larger environment. In essence, bowls can be seen to be part of a wider ecology of surfaces in the home. This notion of surface ecologies-of different surfaces working together and sometimes competing-stands in contrast to the multi-purpose solution of the PC, where an effort is focused on centralizing virtually all operations. Thus, we see the presented sketch as something that should be used only under certain conditions, with a constrained range of operations working in concert with the surroundingsboth physical and social. 


\section{Conclusion}

In this paper, we have shown how an examination of clutter containers in family homes has been used to rethink the management of digital media. The common use of containers like bowls and drawers to manage the litany of stuff that pervades family homes highlights an easily overlooked quality of our everyday domestic experience; it reveals that sometimes, and through perfectly ordinary, unremarkable routines, we find ways of doing just enough to keep the home orderly-of applying minimal effort to keep disorder and clutter at bay [see 23].

Such minimal effort techniques, we have argued, are at odds with the established computing paradigm and, relevant to the presented work, the operations commonly required to store and organize digital media. The dominant PC-centric model demands a level of engagement and attention for transferring media from and to devices, such as cameras, music players, mobile phones, etc., that is far removed from the ease with which objects can be effortlessly placed in containers. The proliferation of media recorders and players for the home has not been matched by a sensitivity towards designing solutions for managing the inevitable growth in volume of media. Instead, efforts have been largely placed on making ever-bigger storage solutions and ever-faster ways to search them. Only the research on visualization techniques has sought to properly address media's proliferation [e.g., 4], and yet this research only succeeds to work within the constraints of the problem, rather than address the fundamental character of the media we capture and store, and our interactions with them.

In our focus on clutter containers, we have attempted to highlight the subtle ways we deal with the material things in our homes. With clutter bowls and junk drawers, we find nuanced ways to keep our homes tidy, to remind ourselves of things we must do, and to sometimes transform the meanings and emotional relations of the objects. Our homes are replete with these simple, embodied practices, so much so that they weave into and bind together the home's social fabric-our homes would not be the same without them.

The sketch of the bowl we have presented is an attempt to explore the need we have to effortlessly handle things. The sketch is also meant to explore how computational resources might be incorporated to build on our intimate familiarity with the physical. On reflection, the sketch has provoked a number of questions that deserve further attention. For example, more thought needs to be given to the functionality envisaged for the bowl. In some respects the mockup appeared to have too many features, encouraging the sorts of prolonged and potentially convoluted interactions we hoped to avoid.

More generally, the presented work has raised a host of questions about digital media containment and possible solutions that address real-world practices in family homes. For instance, the mapping of clutter onto digital media is clearly not a direct one. Further thought needs to be given to the sorts of digital media that might take on clutter-like qualities and consequently what media should and should not be displayed in containers like the augmented bowl we describe. Questions are also raised about the literalness of our interpretations in designing the augmented bowl. Is such a literal translation of physical containers necessary? Also, do such tangible 
interfaces merely limit our potential, while failing to introduce novel and possibly more appealing methods of engaging with our everyday experiences? Why too should our interactions with digital media be constrained by the properties of the physical world when clearly they do not have to?

As we develop the work above and progress towards a fully functioning prototype of an augmented bowl or other container, we hope to address these questions. The position we take here is that detailed studies of established, real-world practices provide a powerful resource in incremental design. This approach is best complimented, however, with in situ prototyping where people's everyday interactions with a solution are used to develop potentially more novel, but still grounded design ideas. What we hope to have done is set the groundwork for a departure from existing notions of storage, changing our expectations of and interactions with digital media.

\section{References}

1. Berg, A.-J. A gendered socio-technical construction: the smart house, in The Social Shaping of Technology, D. MacKenzie and J. Wajcman (eds), (OU Press, Buckingham, 1999), pp. 301-313.

2. Chung, K. H., Oh, K. S., Lee, C. H., Park, J. H., Kim, S., Kim, S. H., Loring, B. and Hass, C. A user-centered approach to designing home network interfaces. Proc. CHI '13, ACM Press, (2003), pp. 648-649.

3. Covington, M. J., Long, W., Srinivasan, S., Dey, A. K., Ahamad, M. and Abowd, G. D. Securing context-aware applications using environment roles. ACM Sympositum on Access Control Models and Technologies, Workshop on Role Based Access Control, ACM Press, (2001), pp. 10-20.

4. Crabtree, A., Hemmings, T. and Rodden, T. Coordinate displays in the home. $C S C W$ ' $(1)$, Workshop on Public, Community and Situated Displays (2002).

5. Crabtree, A. and Rodden, T. Domestic routines and design for the home. Computer Supported Cooperative Work 13(2), (2004), pp. 191-220.

6. Crabtree, A., Rodden, T. and Mariani, J. Collaborating around collections: informing the continued development of photoware. Proc. CSCW' ')4. ACM Press, (2004), pp. 396-405.

7. Douglas, M. Purity and Danger (originally published 1966), (Routledge, London, 2002).

8. Drucker, S.M., Glatzer, A., De Mar, S., Wong, C. SmartSkip: consumer level browsing and skipping of digital video content. Proc. CHI' ()2, ACM Press, (2002), pp. 219-226.

9. Frohlich, D. M. Audiophotography: Bringing Pholos to Life with Sounds (Kluwer Academic, Dordrecht, 2004).

10. Gaver, B. \& Martin, H. Alternatives: exploring information appliances through conceptual design proposals. Proc. CHI'00, ACM Press, (2000), pp. 209-216.

11. Harper, R. (ed) Inside the Smart Home (Springer, London, 2003).

12. Mateas, M., Salvador, T., Scholtz, J., Sorensen, D. Engineering Ethnography in the Home. Proc. CHI '96, ACM Press, (1996), pp. 283-284. 
13. Mungiatapia, E., Intille, S. S. and Larson, K. Activity recognition in the home setting using simple and ubiquitious sensors. Proc. Pervasive '(14, Springer-Verlag, (2004), pp. 158175.

14. Mynatt, E. D., Essa, I. and Rogers, W. Increasing the opportunities for aging in place. Proc. Universal Usability, ACM Press, (2000), pp. 65-71.

15. Norman, D. A. The Psychology of Everyday Things (Basic Books, New York, NY, 1988).

16. O'Brien, J., Rodden, T., Rouncefield, M. and Hughes, J. At home with technology: an ethnographic study of a set-top-box trial. ACM TOCHI 6(3), (1999), pp. 282-308.

17. Rodden, T., Crabtree, A., Hemmings, T., Koleva, B., Humble, J., Åkesson, K.-P. and Hansson, P. Between the dazzle of a new building and its eventual corpse: assembling the ubiquitous home. Proc. DIS '(14, ACM Press, (2004), pp. 71-80.

18. Shen, C., Lesh, N., Vernier, F. Design: Personal digital historian; story sharing around the table. Interactions 10( 2), 2003, pp. 15-22.

19. Spinellis, D. D. The information furnace: consolidated home control. Personal and Ubiquitous Computing 7(1), 2003, pp. 53-69.

20. Suchman, L. Working relations of technology production and use. Computer Supported Cooperative Work 2, (1994), pp. 21-39.

21. Taylor, A. S., Harper, R. Switching on to switch off, in Inside the Smart Home, R. Harper (ed), (Springer-Verlag, London, 2003), pp. 115-126.

22. Taylor, A. S. and Swan, L. Artful systems in the home. Proc. CHI '05, ACM Press, (2005), pp. 641-650.

23. Tolmie, P., Pycock, J., Diggins, T., Maclean, A. and Karsenty, A. Unremarkable computing. Proc. $\mathrm{CHI}$ ')2, ACM Press, (2002), pp. 399-406. 